\title{
VON NEUMANN FACTORS ARE PERFECT
}

\author{
AUBREY WULFSOHN
}

Abstract. It is shown that a von Neumann factor is the $W^{*}$ special associative envelope of its selfadjoint part.

We assume that all algebras, not necessarily associative, have an identity element and that all morphisms are unit preserving. The concepts of associative specialisation and special associative envelope of Jordan algebras have been defined in [3]. An associative specialisation $J \rightarrow A$ is a morphism of a Jordan algebra $J$ into the Jordan algebra consisting of the vector space underlying an associative algebra $A$ provided with the Jordan composition $\frac{1}{2}(a b+b a)$. N. Jacobson [3] calls an algebra with involution perfect if it is the special associative envelope of the Jordan algebra of its symmetric elements. We shall prove that a von Neumann factor is perfect in this sense.

N. Jacobson and C. E. Rickart [4] call a ring $A$, with an identity 1 , a matrix ring $M_{n}$ if $A$ contains a set of matrix units $\left\{e_{i j}\right\}, i, j=1, \cdots, n$, such that $e_{i j} e_{k m}=\delta_{j k} e_{i m}$ and $\sum_{i=1}^{n} e_{i i}=1$. Every element of $A$ can be written in one, and only one, way as $\sum_{i, j} d_{i j} e_{i j}$ where $d_{i j}$ is an element of the subring $D$ of elements of $A$ commuting with all the $e_{i j}$. We shall, to be more specific, denote the matrix ring also by $M_{n}(D)$.

TheOREM (JACOBSON AND RickART [4]). A discrete von Neumann factor is the weak closure of a matrix ring $M_{n}(C)$, for a fixed $n, 1 \leqq n \leqq \infty$. A continuous factor can be realized as a matrix ring $M_{n}(D)$ for any' (finite) $n \geqq 2$.

Definitions. We shall use the term von Neumann algebra to mean abstract von Neumann algebra and morphism of von Neumann algebras to mean normal homomorphism. A Jordan algebra of bounded selfadjoint operators on a Hilbert space is called a J-algebra. Let $A$ denote a von Neumann algebra. We may consider $A$ as an algebra $A_{R}$ over the real field, and we define $A^{+}$to be the vector space underlying $A_{R}$, provided with the weakest operator topology (known to be independent of the realisation of $A)$ and with Jordan composition. A closed Jordan subalgebra of selfadjoint elements of $A^{+}$will be called a $J W$-algebra; so $\mathfrak{h}(A)$, the selfadjoint part

Received by the editors January 22, 1973.

AMS (MOS) subject classifications (1970). Primary 46L10, $17 \mathrm{C} 15$. 
of $A$, is a $J W$-algebra. A weakly closed $J$-algebra will be called a concrete $J W$-algebra. A continuous morphism of $J W$-algebras will be called a $J W$ morphism. A $J W$-morphism from a $J W$-algebra $J$ to $\mathfrak{h}(A)$ is called a $W^{*}$ specialisation of $J$ in $A$. A $W^{*}$-specialisation of a $J W$-algebra $J$ in $\mathscr{L}(H)$, the algebra of all bounded operators in a Hilbert space $H$, is called a $J W$-representation of $J$ in $H$. A pair $(M, \pi)$ consisting of von Neumann algebra $M$ and an associative specialisation of $J$ in $M$ is called a $W^{*}$ envelope of a $J W$-algebra $J$ if, for any $W^{*}$-specialisation $\mu$ of $J$ in a von Neumann algebra $A$, there exists a unique morphism $v$ of $M$ in $A$ such that $\nu \cdot \pi=\mu$. We write $M=\subseteq(J)$. Thus a $J W$-representation of $J$ in $H$ can always be extended to a representation of $\subseteq(J)$ in $H$. Since it is defined by a universal mapping property, $\Xi(J)$ is unique, when it exists.

The mapping $x+i y \rightarrow\left(\begin{array}{cc}x & -y \\ y & x\end{array}\right)$ gives rise to an injective functor $\tau$ of the category of complex algebras of operators, in complex Hilbert spaces, to the category of real algebras of operators, in real Hilbert spaces. Every element of a concrete von Neumann algebra $A$ can be written as a matrix possibly infinite (see $[2,1.2]$ ), and $\tau(x)$ is the matrix where each entry of the matrix of $x \in A$ is replaced by the corresponding $2 \times 2$ matrix. If $x$ is hermitian then $\tau(x)$ will be symmetric. We may identify the algebras $\tau(A)$ and $A_{R}$. We shall call $\tau$-algebras the images of the complex algebras and call $\tau$-morphism a morphism of the real algebras such that the image of a $\tau$-algebra is a $\tau$-algebra. The category of concrete $\tau$-von Neumann algebras with normal $\tau$-homomorphisms is clearly equivalent to the category of concrete von Neumann algebras with normal homomorphisms.

Jacobson and Rickart [4] show that, for $n \geqq 2$, the associative ring enveloping $\mathfrak{h}\left(M_{n}\right)$, the Jordan ring of selfadjoint elements of $M_{n}$, is the matrix ring $M_{n}$ itself, and also that if every $x \in \mathfrak{h}\left(M_{n}\right)$ is of the form $a+a^{*}, a \in M_{n}$, then for $n \geqq 3$ the special associative envelope of $\mathfrak{h}\left(M_{n}\right)$ is $M_{n}$ itself. We use the same technique to show that $M=\Xi(h(M))$ whenever $M$ is a von Neumann factor.

Let $M$ be a von Neumann factor and suppose $J=\mathfrak{h}(M)$. There exists a family $\left\{e_{i}\right\}_{i \in K}$ of orthogonal, nonzero, equivalent projections with $\bigoplus_{i \in K} e_{i}=1$ (the sum is the l.u.b. in the weakest operator topology of the increasing directed set of finite sums). These $e_{i}$ are the $e_{i i}$ of the matrix ring realisation of $M$. We call the above sum a partition of the identity for $M$.

Proposition 1. Let $M$ be a von Neumann factor and let e be an element of a partition of the identity for $M$, the index set $K$ having cardinality $\geqq 3$. Then $M e M=M$.

Proof. It is obvious that $M e M \subset M$. We consider $M$ as the closure of a matrix ring and assume that $e=e_{k \xi}$. Any element of $M$ may be approached, 
in the weak operator topology, by $\sum_{i, j} d_{i j} e_{i j}$, and $d_{i j} e_{i j}=d_{i j} e_{i k} e_{k k} e_{k j} \in M e M$. Thus, since $M$ and $M e M$ are weakly closed, $M=M e M$.

Proposition 2 (The Peirce decomposition of a von Neumann factor). Let $M$ be a von Neumann factor with partition of the identity $\left\{e_{i}\right\}_{i \in K}$, the index set $K$ having cardinality $\geqq 3$. For $i, j \in K$, let $M_{i j}=e_{i} M e_{j}$. Then

$$
\begin{aligned}
M_{i j} M_{m n} & =0 & & \text { if } j \neq m, \\
& =M_{i n} & & \text { if } j=m, i \neq n, \\
& =M_{i k} M_{k i} & & \text { for all } k \neq i, \text { whenever } i=n \neq j=m .
\end{aligned}
$$

(ii) The $M_{i j}$ are weakly closed subspaces of $M$.

(iii) $M_{i i}$ is the von Neumann algebra generated by $M_{i k} M_{k i}$ (which is independent of $k$ by (i)).

(iv) Every element of $M$ is the weak limit of sums of elements of the $M_{i j}$.

Proof. (i) is known for (two-sided) Peirce decompositions of associative algebras, (ii) $M_{i j}$ is weakly closed since, if $e_{i} a^{(p)} e_{j}$ converges to $a$ weakly, then $a=e_{i} a e_{j} \in M_{i j}$. (iii) Clearly $M_{i k} M_{k i}$ is contained in $M_{i i}$ and is closed under multiplication. Using Proposition 1, we see that $M_{i i} \subset M_{i k} M_{k i}$. (iv) is clear since any $x \in M$ can be approximated in the weakest operator topology by $\left(\bigoplus_{i \in I} e_{i}\right) x\left(\bigoplus_{i \in I} e_{i}\right)$, where $I$ covers all finite subsets of $K$.

THEOREM 1. Let $J$ be the selfadjoint part of a concrete von Neumann factor $M$, where we assume that $M \neq C$. For any $W^{*}$-specialisation of $J$ in a von Neumann algebra $A$, there is a unique normal homomorphism of $M_{R}$ in $A_{R}$.

Proof. For $M$ of type $\mathrm{I}_{2}$ the result is known (cf. [1, Table 1, $k=3$ ]). So we shall assume that $M$ is neither type $\mathrm{I}_{1}$ nor type $\mathrm{I}_{2}$. Suppose the partition of the identity for $M$ is $\left\{e_{i}\right\}$. Let $M_{i j}=e_{i} M e_{j}$ and $J_{i j}=J \cap\left(M_{i j}+M_{j i}\right)$. Let $\mu$ be a $W^{*}$-specialisation of $J$ in $A$ and denote $\mu(x)$ as $\bar{x}$, for $x \in J$. Then we have a Peirce decomposition of $A$ with $A_{i j}=\bar{e}_{i} A \bar{e}_{j}$. In fact it is easily verified that $\left\{\bar{e}_{i}\right\}$ is a decomposition of the identity of $A$; we do not need the properties (iii) and (iv) of Proposition 2.

Assume $i \neq j$. It is easily seen that every element of $J_{i j}$ is of the form $x_{i j}=e_{i} x e_{j}+e_{j} x^{*} e_{i}$, where $x \in M$, that

$$
\bar{x}_{i j}=\mu\left(x_{i j}\right)=\mu\left(e_{i} x_{i j} e_{j}+e_{j} x_{i j} e_{i}\right)=\bar{e}_{i} \bar{x}_{i j} \bar{e}_{j}+\bar{e}_{j} \bar{x}_{i j} \bar{e}_{i}
$$

and that this expression of $\mu\left(\bar{x}_{i j}\right)$ in $A_{i j}+A_{j i}$ is unique. Thus we can define a linear map of $M_{i j}$ into $A_{i j}$ by $v_{i j}\left(e_{i} x e_{j}\right)=\bar{e}_{i} \bar{x}_{i j} \bar{e}_{j}$. It is also bounded. In fact $\left\|\bar{x}_{i j}\right\| \leqq \beta\left\|x_{i j}\right\| \leqq 2 \beta\left\|e_{i} x e_{j}\right\|$ for some $\beta<+\infty$ and thus $\left\|\bar{e}_{i} \bar{x}_{i j} \bar{e}_{j}\right\| \leqq$ $2 \beta\left\|\bar{e}_{i}\right\|\left\|\bar{e}_{j}\right\|\left\|e_{i} x e_{j}\right\|$. As in [5], using a third index $k \neq i, j$, we can show that there exists a unique linear map $v_{i i}^{\prime}$ of $M_{i j} M_{j i}$ into $A_{i i}$ such that $v_{i i}^{\prime}(a b)=v_{i j}^{\prime}(a) v_{j i}^{\prime}(b)$ for $a \in M_{i j}, b \in M_{j i}$ and that $v_{i i}^{\prime}$ is independent of the 
choice of $j$. It is clear that $\boldsymbol{v}_{i i}^{\prime}$ is bounded, multiplicative and that it extends to a real homomorphism $v_{i i}$. We can define a bounded linear map $v^{\prime \prime}$ of $\bigoplus_{m, n} M_{m n}$ into $A$ which coincides with $v_{m n}$ on $M_{m n}$. The same procedure as in [5] can be used to show that the relation $v^{\prime \prime}(a b)=v^{\prime \prime}(a) v^{\prime \prime}(b)$ holds for $a \in M_{m n}, b \in M_{r s}$, for any $m, n, r, s$, so $v^{\prime \prime}$ extends to a homomorphism $v$ of $M_{R}$ into $A_{R}$. Moreover, $v$ is a normal homomorphism. In fact, since $\mu$ is $W^{*}$-continuous, the sequence of elements $\bar{x}_{m n}^{(p)}=\mu\left(e_{m} x^{(p)} e_{n}+e_{n} x^{(p) *} e_{m}\right)$ converges to $\bar{x}_{m n}$ in $A_{m n}$ whenever $x^{(p)}$ converges to $x$ in $M$, the convergence being with respect to the weakest operator topologies of $A$ and $M$. Since the $\bar{x}_{i j}$ are selfadjoint, it is evident that $v$ is $a^{*}$-homomorphism. that

The map $\pi$ is here an immersion, so to see that $\nu \cdot \pi=\mu$ we must show

$$
\text { and } \quad \begin{gathered}
\mu\left(x_{i j}\right)=v_{i j}\left(e_{i} x e_{j}\right)+v_{j i}\left(e_{j} x^{*} e_{i}\right) \\
\mu\left(x_{i j} x_{j i}\right)=v_{i j}\left(e_{i} x e_{j}\right) v_{j i}\left(e_{j} x e_{i}\right)+v_{j i}\left(e_{j} x^{*} e_{i}\right) v_{i j}\left(e_{i} x^{*} e_{j}\right) .
\end{gathered}
$$

This can be done as in [5]. To prove $v$ unique we show that $M$ is the von Neumann algebra generated by $J$. In fact, $M$ is the von Neumann algebra generated by the elements of the $M_{i j}, i \neq j$, and for $i \neq j, x_{i j} e_{j}=e_{i} x e_{j} \in M_{i j}$.

THEOREM 2. $\Xi(J)=M$ whenever $J$ is the selfadjoint part of a factor $M$, where we exclude the case where $M$ has a realisation as $\boldsymbol{C}$.

Proof. Since the $W^{*}$-envelope is unique it is sufficient to prove the theorem for a concrete factor. Since, in Theorem $1, v\left(e_{i} x e_{j}\right)=\bar{e}_{i} \bar{x}_{i j} \bar{e}_{j}$, it is evident that $v$ is a $\tau$-morphism, so the theorem follows from Theorem 1.

\section{BIBLIOGRAPHY}

1. M. F. Atiyah, R. Bott and A. Shapiro, Clifford modules, Topology 3 (1964), suppl. 1, 3-38. MR 29 \#5250.

2. J. Dixmier, Les algèbres d'opérateurs dans l'espace hilbertien, Cahiers scientifiques, fasc. 25, Gauthier-Villars, Paris, 1957. MR 20 \#1234.

3. N. Jacobson, Structure and representations of Jordan algebras, Amer. Math. Soc. Colloq. Publ., vol. 39, Amer. Math. Soc., Providence, R.I., 1968. MR 40 \#4330.

4. N. Jacobson and C. E. Rickart, Homomorphisms of Jordan rings of self-adjoint elements, Trans. Amer. Math. Soc. 72 (1952), 310-322. MR 13, 719.

5. W. S. Martindale III, Jordan homomorphisms of the symmetric elements of a ring with involution, J. Algebra 5 (1967), 232-249. MR 35 \#1636.

Centre de Recherches Mathematiques, Université de Montréal, Montréal, Québec, Canada 